CIRJE-F-777

\title{
Hedging European Derivatives with the Polynomial Variance Swap under Uncertain Volatility Environments
}

\author{
Akihiko Takahashi \\ University of Tokyo \\ Yukihiro Tsuzuki \\ Mizuho-DL Financial Technology Co., Ltd. \\ Akira Yamazaki \\ Graduate School of Economics, University of Tokyo
}

December 2010

CIRJE Discussion Papers can be downloaded without charge from:

http://www.cirje.e.u-tokyo.ac.jp/research/03research02dp.html

Discussion Papers are a series of manuscripts in their draft form. They are not intended for circulation or distribution except as indicated by the author. For that reason Discussion Papers may not be reproduced or distributed without the written consent of the author. 


\title{
Hedging European Derivatives with the Polynomial Variance Swap under Uncertain Volatility Environments *
}

\author{
Akihiko Takahashi† Yukihiro Tsuzuki ${ }^{\ddagger}$ Akira Yamazaki ${ }^{\S}$
}

December 7, 2010

\begin{abstract}
This paper proposes a new hedging scheme of European derivatives under uncertain volatility environments, in which a weighted variance swap called the polynomial variance swap is added to the Black-Scholes delta hedging for managing exposure to volatility risk. In general, under these environments one cannot hedge the derivatives completely by using dynamic trading of only an underlying asset owing to volatility risk. Then, for hedging uncertain volatility risk, we design the polynomial variance, which can be dependent on the level of the underlying asset price. It is shown that the polynomial variance swap is not perfect, but more efficient as a hedging tool for the volatility exposure than the standard variance swap. In addition, our hedging scheme has a preferable property that any information on the volatility process of the underlying asset price is unnecessary. To demonstrate robustness of our scheme, we implement Monte Carlo simulation tests with three different settings, and compare the hedging performance of our scheme with that of standard dynamic hedging schemes such as the minimum-variance hedging. As a result, it is found that our scheme outperforms the others in all test cases. Moreover, it is noteworthy that the scheme proposed in this paper continues to be robust against model risks.
\end{abstract}

Keywords: European Derivatives, Black-Scholes Delta Hedging, Uncertain Volatility Risk, Polynomial Variance Swap

\footnotetext{
* Forthcoming in International Journal of Theoretical and Applied Finance. This research is supported by CARF (Center for Advanced Research in Finance) and the global COE program "The research and training center for new development in mathematics." All the contents expressed in this research are solely those of the authors and do not represent the view of any institutions. The authors are not responsible or liable in any manner for any losses and/or damages caused by the use of any contents in this research.

${ }^{\dagger}$ Graduate School of Economics, University of Tokyo

${ }^{\ddagger}$ Mizuho-DL Financial Technology Co., Ltd.

$\S$ Graduate School of Economics, University of Tokyo, Mizuho-DL Financial Technology Co., Ltd.
} 


\section{Introduction}

This paper proposes a new hedging scheme of European derivatives under uncertain volatility environments, in which an exotic variance swap called the polynomial variance swap is added to the Black-Scholes delta hedging in order to hedge volatility risk. To examine robustness of our hedging scheme, we implement Monte Carlo simulation tests with three different settings of underlying processes and compare the hedging performance of our scheme with that of other standard hedging schemes.

Variance swaps, which pay the realized variance of the returns on an underlying price process and receive the fixed positive amount, have become the most approved tools for trading volatility. Moreover, with a remarkable development of volatility derivatives both in practice and academically, various types of derivatives on realized variance/volatility are proposed; for examples, corridor variance swaps (Carr and Lewis [2004]), gamma swaps (Mougeot [2005]), conditional variance swaps (Mougeot [2005]), moment swaps (Schoutens [2005]), volatility swaps (Carr and Lee [2008], and Friz and Gatheral [2005]), multi-asset stochastic local variance contracts (Carr and Laurence [2009]), and options on realized variance/volatility (Carr et al. [2005], and Carr and Lee [2007, 2009]). By virtue of much energetic research, institutions can deal with a large number of volatility-based trading strategies, and have invested in such realized variance/volatility contracts as new asset classes.

On the other hand, our interest is how robust the Black-Scholes delta hedging is under uncertain volatility environments when adding a certain variance swap. It is widely recognized that the variance swap is a useful tool for managing exposure to volatility risk. This recognition, however, is questionable. For example, volatility exposure of a call option is higher at-the-money than out/inthe-money, while the standard variance swap has invariant volatility exposure with respect to the underlying asset price. The similar problem exists with respect to time-to-maturity. In general, it can be said that there is a discrepancy between volatility risks of the hedging target derivative and the variance swap, and volatility exposure of the variance swap cannot be completely balanced with that of the derivative security. Therefore, the standard variance swap might not work effectively to hedge volatility risk. This detailed discussion is stated in the next section.

To overcome this problem, a special realized variance/volatility contract for hedging volatility exposure on European derivatives is needed, and it is necessary to consider a new hedging scheme using this contract. However, to the best of our knowledge, there is no research designing such a variance/volatility contract and proposing such a hedging strategy. Besides, in the past literature numerical experiment or empirical analysis, which examines the hedging performance in the case that the standard variance swap is used as a hedging tool of volatility exposure, is very limited.

The purpose of this paper is as follows: First, we design a weighted variance swap called the polynomial variance swap for hedging volatility exposure on path-independent European derivatives. Second, we propose a new robust hedging scheme against exposure to volatility risk, in which the polynomial variance swap is added to the Black-Scholes delta hedging. Third, through Monte Carlo simulation tests, we confirm effectiveness of our hedging scheme. In contrast to the hedging schemes with uncertain/stochastic volatilities proposed in the past literature (e.g., Avellaneda et al. [1995], Bakshi et al. [1997], Heath et al. [2001a, 2001b], Fink [2003], and Takahashi and Yamazaki [2009b]), our scheme has a preferable property that any information about volatility process of the underlying asset is unnecessary. Therefore, it can be expected that our 
hedging scheme minimizes model risk and continues to be robust to the hedging performance under any uncertain volatility environments.

The rest of this paper is organized as follows: In the next section, we refer to the hedging error due to uncertainty of volatility when applying the BlackScholes delta hedging. Then, it well be shown that the standard variance swap is not entirely appropriate to hedge exposure to volatility risk. The third section introduces the polynomial variance swap as a hedging tool for uncertain volatility risk. The polynomial variance swap admits model-free replication. The fourth section provides numerical experiments for verifying effectiveness of our hedging scheme. As a result, it is found that our scheme is more robust than other standard hedging schemes under uncertain volatility environments. Finally, we give concluding remarks in the last section. The proofs of some propositions are stated in appendix.

\section{Hedging Uncertain Volatility Risk}

In this section, we briefly review the hedging error caused by uncertain volatility risk when using the Black-Scholes delta hedging. Then, we consider applying the standard variance swap to eliminate this error. However, it will be demonstrated that the standard variance swap is not quite a suitable tool for hedging uncertain volatility risk.

\subsection{Uncertain Volatility Risk on European Derivatives}

We assume a frictionless and no-arbitrage market. Let $S_{t}$ be the spot price of a certain stock, an underlying asset price at time $t \in\left[0, T^{*}\right]$ where $T^{*}$ is some arbitrarily determined time horizon. For simplicity, both the risk-free interest rate and the dividend yield of the stock are assumed to be zero. The no-arbitrage assumption ensures the existence of a risk-neutral probability measure $\mathbb{Q}$ such that the instantaneous expected rate of return on every asset is equal to the instantaneous interest rate; i.e., it is equal to zero in our setting. Furthermore, the risk-neutral process of the underlying asset price is assumed to be an Itô process under a filtered probability space $\left(\Omega, \mathcal{F},\left\{\mathcal{F}_{t}\right\}_{t \in\left[0, T^{*}\right]}, \mathbb{Q}\right)$. Thus the underlying asset price $S$ under the risk-neutral measure $\mathbb{Q}$ is given by the unique solution of the following stochastic differential equation (SDE):

$$
d S_{t}=\sigma(\omega, t) S_{t} d W_{t},
$$

where $W$ is a Brownian motion under $\mathbb{Q}$, and $\sigma$ is a $\mathbf{R}$-valued $\left\{\mathcal{F}_{t}\right\}$-progressively measurable process that guarantees that unique solution to SDE (2.1).

Suppose that $f^{T}(S)$ is the payoff at maturity $T$ of a path-independent European derivative whose randomness depends solely on the underlying asset price at maturity, $S_{T}$. If a trader hedges the derivative security over the period by the Black-Scholes delta hedging with a fixed volatility $\sigma_{\mathrm{H}}>0$ called the hedging volatility, then it holds the following equation:

$$
\begin{aligned}
f^{T}\left(S_{T}\right)= & v\left(0, S_{0} ; \sigma_{\mathrm{H}}, f^{T}\right)+\int_{0}^{T} \frac{\partial v}{\partial S}\left(t, S_{t} ; \sigma_{\mathrm{H}}, f^{T}\right) d S_{t} \\
& +\frac{1}{2} \int_{0}^{T} S_{t}^{2} \frac{\partial^{2} v}{\partial S^{2}}\left(t, S_{t} ; \sigma_{\mathrm{H}}, f^{T}\right)\left[\sigma^{2}(\omega, t)-\sigma_{\mathrm{H}}^{2}\right] d t
\end{aligned}
$$

where $v\left(t, S_{t} ; \sigma_{\mathrm{H}}, f^{T}\right)$ denote the Black-Scholes price function with the constant volatility $\sigma_{\mathrm{H}}$ and payoff $f^{T}$ at time $t$, and $\frac{\partial v}{\partial S}\left(t, S_{t} ; \sigma_{\mathrm{H}}, f^{T}\right)$ and $\frac{\partial^{2} v}{\partial S^{2}}\left(t, S_{t} ; \sigma_{\mathrm{H}}, f^{T}\right)$ are the Black-Scholes delta and gamma, respectively. The first term on the 
right hand side of Eq.(2.2) is the premium of the derivative security, and the second term is the dynamic portfolio by the Black-Scholes delta with the hedging volatility $\sigma_{\mathrm{H}}$. The third term,

$$
\mathrm{HE}:=\frac{1}{2} \int_{0}^{T} S_{t}^{2} \frac{\partial^{2} v}{\partial S^{2}}\left(t, S_{t} ; \sigma_{\mathrm{H}}, f^{T}\right)\left[\sigma^{2}(\omega, t)-\sigma_{\mathrm{H}}^{2}\right] d t,
$$

is the hedging error caused by uncertain volatility risk, which depends on the difference between the instantaneous volatility $\sigma(\omega, t)$ and the hedging volatility $\sigma_{\mathrm{H}}$. If $\sigma(\omega, t)$ is completely predictable for all $t \in[0, T]$, the hedging error $\mathrm{HE}$ can be eliminated perfectly. However, this situation is obviously unrealistic and there exists uncertainty of volatilities in a real market all the time. In this paper, we explore that how the Black-Scholes delta hedging can be improved by using the variance swap under uncertain volatility environments.

\subsection{Hedging Volatility Risk with Variance Swap}

The hedging error HE can be decomposed into

$$
\mathrm{HE}=\int_{0}^{T} g\left(t, S_{t}\right) \sigma^{2}(\omega, t) d t-\sigma_{\mathrm{H}}^{2} \int_{0}^{T} g\left(t, S_{t}\right) d t=: A-B,
$$

where

$$
g\left(t, S_{t}\right):=\frac{1}{2} S_{t}^{2} \frac{\partial^{2} v}{\partial S^{2}}\left(t, S_{t} ; \sigma_{\mathrm{H}}, f^{T}\right)
$$

and

$$
A:=\int_{0}^{T} g\left(t, S_{t}\right) \sigma^{2}(\omega, t) d t, \quad B:=\sigma_{\mathrm{H}}^{2} \int_{0}^{T} g\left(t, S_{t}\right) d t .
$$

In the following, $g(t, S)$ is called the volatility risk weight on the derivative security at time $t$ and stock price $S$. In Section 2.2 .1 and 2.2.2, we consider the hedging schemes of risk $A$ and risk $B$ in Eq.(2.4), separately.

\subsubsection{Hedging Risk B}

Firstly, we present a hedging scheme of risk $B$. By regarding $g^{t}(S):=g(t, S)$ as a payoff function at maturity $t$, the volatility risk weight can be represented in the following form:

$$
\begin{aligned}
g\left(t, S_{t}\right)= & v\left(0, S_{0} ; \sigma_{\mathrm{H}}, g^{t}\right)+\int_{0}^{t} \frac{\partial v}{\partial S}\left(u, S_{u} ; \sigma_{\mathrm{H}}, g^{t}\right) d S_{u} \\
& +\frac{1}{2} \int_{0}^{t} S_{u}^{2} \frac{\partial^{2} v}{\partial S^{2}}\left(u, S_{u} ; \sigma_{\mathrm{H}}, g^{t}\right)\left[\sigma^{2}(\omega, u)-\sigma_{\mathrm{H}}^{2}\right] d u,
\end{aligned}
$$

for $0 \leq u \leq t$. The third term on the right hand side of Eq.(2.7) is the hedging error of risk $B$; i.e., the hedging error of the hedging error HE. This error, however, is negligible because it is much smaller than HE in usual. Therefore, by time-discretization and neglecting the hedging error of risk $B$, it satisfies

$$
B \approx \sigma_{\mathrm{H}}^{2} \int_{0}^{T} v\left(0, S_{0} ; \sigma_{\mathrm{H}}, g^{t}\right) d t+\sum_{n=1}^{N} \sigma_{\mathrm{H}}^{2} \Delta t \int_{0}^{t_{n}} \frac{\partial v}{\partial S}\left(u, S_{u} ; \sigma_{\mathrm{H}}, g^{t_{n}}\right) d S_{u},
$$

where $t_{n}=n \Delta t$ is the re-balance timing of the dynamic hedging and $t_{N}=T$. Note that $\sigma_{\mathrm{H}}^{2} \int_{0}^{T} v\left(0, S_{0} ; \sigma_{\mathrm{H}}, g^{t}\right) d t$ is the initial cost of risk $B$ and the second term on the right hand side of Eq.(2.8) is the dynamic portfolio for hedging risk $B$. The closed-form expressions of $v\left(u, S_{u} ; \sigma_{\mathrm{H}}, g^{t}\right)$ and $\frac{\partial v}{\partial S}\left(u, S_{u} ; \sigma_{\mathrm{H}}, g^{t}\right)$ can be found in Appendix A. 


\subsubsection{Hedging Risk A by Using Standard Variance Swap}

Secondly, we consider a hedging scheme of risk $A$ by using the standard variance swap. The variance swap is now actively traded in over-the-counter (OTC) on both stocks and stock indices. In this contract, one party agrees with the other to receive the realized variance of returns of a specified underlying asset over a specified future period. In return, the party pays a fixed positive amount at expiry. The fixed positive amount is agreed upon at the initial time and chosen so that the variance swap is costless to enter. That is, the variance swap in continuous-time setting has the following payoff:

$$
M\left\{\frac{1}{T_{\mathrm{VS}}} \int_{0}^{T_{\mathrm{VS}}} \sigma^{2}(\omega, t) d t-K_{\mathrm{VS}}\right\},
$$

where $M, K_{\mathrm{VS}}$, and $T_{\mathrm{VS}}$ are a notional amount, a fixing rate, and maturity of the variance swap, respectively. Thus, in the contract (2.9) the party receives $\frac{M}{T_{\mathrm{VS}}} \int_{0}^{T_{\mathrm{VS}}} \sigma^{2}(\omega, t) d t$ while he pays $M K_{\mathrm{VS}}$.

It is well-known that the floating side of the variance swap, which is the realized variance of an underlying asset price, admits model-free replication by a static position in options and dynamic trading of the underlying asset; and the fixing rate $K_{\mathrm{VS}}$ is determined by the initial value of the static position. See Derman et al. [1999] and Carr and Madan [1998] for details of the standard variance swap.

Consider the situation in which a trader tries to hedge risk $A$ by using the variance swap. To do this, it is necessary to choose an appropriate notional amount of the variance swap. If he can choose the notional amount $M=g\left(t, S_{t}\right) T_{\mathrm{VS}}$ for all $t \in[0, T]$ and $S_{t}>0$, then risk $A$ is entirely fixed to $M K_{\mathrm{VS}}$. In addition, by setting the hedging volatility $\sigma_{\mathrm{H}}=\sqrt{K_{\mathrm{VS}}}$, the initial cost for hedging risk $B$ in Eq.(2.8) can be balanced with the fixed payment of the variance swap, $M K_{\mathrm{VS}}$. By virtue of the standard variance swap, a robust hedging scheme for uncertain volatility risk is apparently able to be implemented. However, this scheme seems to be not successful because the volatility risk weight $g\left(t, S_{t}\right)$ highly depends on both time $t$ and the underlying asset price $S_{t}$ in general. Figure 1 shows over/under-hedging situations when using the variance swap as a hedging tool of risk $A$ on a call with strike $K=100$ and maturity $T=1$. In order to improve this problem, we develop a new variance swap called the polynomial variance swap in the next section.

\section{Polynomial Variance Swap}

In this section we introduce the polynomial variance swap (PVS) for hedging uncertain volatility risk of European derivatives, instead of the standard variance swap. PVS has the following payoff:

$$
\int_{0}^{T_{\mathrm{PVS}}} \mathbf{1}_{\left\{S_{t} \in I\right\}} P_{M}\left(S_{t}\right) \sigma^{2}(\omega, t) d t-K_{\mathrm{PVS}},
$$

where $I=[a, b], 0 \leq a<b$ denotes a corridor interval, $T_{\mathrm{PVS}}$ is maturity, $K_{\mathrm{PVS}}$ is a fixed payment of PVS, and $P_{M}(x)$ is the $M$-th order polynomial, that is,

$$
P_{M}(x):=a_{0}+a_{1} x+a_{2} x^{2}+\cdots+a_{M} x^{M} .
$$

Here, $a_{0}, a_{1}, \cdots, a_{M} \in \mathbf{R}$ are coefficients of $P_{M}(x)$. Note that PVS is a generalization of some variations on variance swaps which several institutes have offered; e.g., the standard variance swap, the corridor variance swap, and the 
gamma swap. By a suitable choice of the order and the coefficients of the polynomial, PVS allows us an arbitrary allocation of the volatility risk weight with respect to the underlying asset price.

\subsection{Replication of Polynomial Variance}

Similarly to the standard variance swap, PVS admits model-free replication by a static portfolio in options and dynamic trading of the underlying asset. In this subsection the replication scheme of PVS is provided.

Note that PVS is a linear combination of power variances with a corridor:

$$
\int_{0}^{T_{\mathrm{PVS}}} \mathbf{1}_{\left\{S_{t} \in I\right\}} S_{t}^{m} \sigma^{2}(\omega, t) d t .
$$

Hence it is sufficient to demonstrate the model-free replication scheme of each power variance.

First, in the case of $m=0$, the power variance swap is obviously equivalent to the corridor variance swap introduced by Carr and Lewis [2004].

Proposition 1 Let $I=[a, b]$ be an interval. Define $S_{t}^{*}=\max \left(a, \min \left(S_{t}, b\right)\right)$. Then, for any $\kappa \in I$ and all $T \in\left[0, T^{*}\right]$, it satisfies

$$
\begin{aligned}
\int_{0}^{T} \mathbf{1}_{\left\{S_{t} \in I\right\}} \sigma^{2}(\omega, t) d t & =\int_{a}^{\kappa} \frac{2}{K^{2}}\left(K-S_{T}\right)^{+} d K \\
& +\int_{\kappa}^{b} \frac{2}{K^{2}}\left(S_{T}-K\right)^{+} d K \\
& -2\left\{\ln \frac{\kappa}{S_{0}^{*}}+\frac{S_{0}}{\kappa}-\frac{S_{0}}{S_{0}^{*}}\right\} \\
& -2 \int_{0}^{T}\left(\frac{1}{\kappa}-\frac{1}{S_{t}^{*}}\right) d S_{t} .
\end{aligned}
$$

Proof: See Carr and Lewis [2004].

The first and second term on the right hand side of Eq.(3.4) are put and call static portfolios, respectively. Moreover, the fourth term is a dynamic portfolio of the underlying asset. Note that all of the portfolios in Eq.(3.4) are model-free.

Next, the following proposition shows the replication portfolio of power variance in the case of $m=1$.

Proposition 2 Let $I=[a, b]$ be an interval. Define $S_{t}^{*}=\max \left(a, \min \left(S_{t}, b\right)\right)$. Then, for any $\kappa \in I$ and all $T \in\left[0, T^{*}\right]$, it satisfies

$$
\begin{aligned}
\int_{0}^{T} \mathbf{1}_{\left\{S_{t} \in I\right\}} S_{t} \sigma^{2}(\omega, t) d t & =\int_{a}^{\kappa} \frac{2}{K}\left(K-S_{T}\right)^{+} d K \\
& +\int_{\kappa}^{b} \frac{2}{K}\left(S_{T}-K\right)^{+} d K \\
& -2\left\{S_{0} \ln \frac{S_{0}^{*}}{\kappa}-S_{0}^{*}+\kappa\right\} \\
& -2 \int_{0}^{T} \ln \frac{S_{t}^{*}}{\kappa} d S_{t} .
\end{aligned}
$$

Proof: See Appendix B. 
Note that all of the portfolios on the right hand side of Eq.(3.5), which are static option positions and a dynamic position consisting of the underlying asset, are also model-free.

Finally, the following proposition presents the replication portfolio of power variance in the case of $m \geq 2$.

Proposition 3 Let $I=[a, b]$ be an interval and $m \geq 2$ be an integer. Define $S_{t}^{*}=\max \left(a, \min \left(S_{t}, b\right)\right)$. Then, for any $\kappa \in I$ and all $T \in\left[0, T^{*}\right]$, it satisfies

$$
\begin{aligned}
& \int_{0}^{T} \mathbf{1}_{\left\{S_{t} \in I\right\}} S_{t}^{m} \sigma^{2}(\omega, t) d t \\
& =\int_{a}^{\kappa} 2 K^{m-2}\left(K-S_{T}\right)^{+} d K \\
& +\int_{\kappa}^{b} 2 K^{m-2}\left(S_{T}-K\right)^{+} d K \\
& -\frac{2}{m(m-1)}\left\{(1-m)\left(S_{0}^{*}\right)^{m}-m S_{0}\left[\kappa^{m-1}-\left(S_{0}^{*}\right)^{m-1}\right]-(1-m) \kappa^{m}\right\} \\
& -\frac{2}{m-1} \int_{0}^{T}\left[\left(S_{t}^{*}\right)^{m-1}-\kappa^{m-1}\right] d S_{t} .
\end{aligned}
$$

Proof: See Appendix C.

Similarly to the case of $m=0$ and 1 , all of the portfolios on the right hand side of Eq.(3.6) are model-free.

\subsection{Strike Volatility of Polynomial Variance Swap}

When giving a polynomial $P_{M}(x)$ and a corridor $I=[a, b]$, the fixed payment $K_{\mathrm{PVS}}$ of PVS can be computed easily as follows:

$$
K_{\mathrm{PVS}}:=\mathbb{E}\left[\int_{0}^{T_{\mathrm{PVS}}} \mathbf{1}_{\left\{S_{t} \in I\right\}} P_{M}\left(S_{t}\right) \sigma^{2}(\omega, t) d t\right]=\sum_{m=0}^{M} a_{m} \beta_{m},
$$

where $\mathbb{E}[\cdot]$ denotes the expectation operator under the risk-neutral measure $\mathbb{Q}$, $a_{0}, a_{1}, \cdots, a_{M} \in \mathbf{R}$ are coefficients of $P_{M}(x)$,

$$
\begin{aligned}
\beta_{0} & :=\mathbb{E}\left[\int_{0}^{T_{\mathrm{PVS}}} \mathbf{1}_{\left\{S_{t} \in I\right\}} \sigma^{2}(\omega, t) d t\right] \\
& =\int_{a}^{S_{0}} \frac{2}{K^{2}} P(K, T) d K+\int_{S_{0}}^{b} \frac{2}{K^{2}} C(K, T) d K, \\
\beta_{1} & :=\mathbb{E}\left[\int_{0}^{T_{\mathrm{PVS}}} \mathbf{1}_{\left\{S_{t} \in I\right\}} S_{t} \sigma^{2}(\omega, t) d t\right] \\
& =\int_{a}^{S_{0}} \frac{2}{K} P(K, T) d K+\int_{S_{0}}^{b} \frac{2}{K} C(K, T) d K,
\end{aligned}
$$

and when $m \geq 2$,

$$
\begin{aligned}
\beta_{m} & :=\mathbb{E}\left[\int_{0}^{T_{\mathrm{PVS}}} \mathbf{1}_{\left\{S_{t} \in I\right\}} S_{t}^{m} \sigma^{2}(\omega, t) d t\right] \\
& =\int_{a}^{S_{0}} 2 K^{m-2} P(K, T) d K+\int_{S_{0}}^{b} 2 K^{m-2} C(K, T) d K .
\end{aligned}
$$


Here, $P(K, T)$ and $C(K, T)$ represent the time-0 prices of plain vanilla put and call options with spot price $S_{0}$, strike $K$ and maturity $T$, respectively.

On the other hand, the strike volatility $\sigma_{\mathrm{PVS}}$ of PVS is defined as a positive constant such that

$$
\mathbb{E}\left[\int_{0}^{T_{\mathrm{PVS}}} \mathbf{1}_{\left\{S_{t} \in I\right\}} P_{M}\left(S_{t}\right) \sigma^{2}(\omega, t) d t\right]=\mathbb{E}\left[\int_{0}^{T_{\mathrm{PVS}}} \mathbf{1}_{\left\{S_{t} \in I\right\}} P_{M}\left(S_{t}\right) \sigma_{\mathrm{PVS}}^{2} d t\right]
$$

In the simulation analysis, we adopt the strike volatility $\sigma_{\mathrm{PVS}}$ as the hedging volatility of the Black-Scholes delta hedging with/without PVS. The strike volatility is computed by the following proposition:

Proposition 4 Let $I=[a, b]$ be an interval. Then, the strike volatility $\sigma_{P V S}$ is given by

$$
\sigma_{P V S}=\sqrt{\frac{K_{P V S}}{L_{P V S}}}
$$

where $K_{P V S}$ is defined in Eq.(3.7), and

$$
\begin{aligned}
L_{P V S} & :=\int_{0}^{T_{P V S}} P_{M}(\kappa) \mathbb{E}\left[\mathbf{1}_{\left\{S_{t} \in I\right\}}\right] d t+\int_{0}^{T_{P V S}} P_{M}^{\prime}(\kappa) \mathbb{E}\left[\mathbf{1}_{\left\{S_{t} \in I\right\}}\left(S_{t}-\kappa\right)\right] d t \\
& +\int_{0}^{T_{P V S}} \int_{0}^{\kappa} P_{M}^{\prime \prime}(K) \mathbb{E}\left[\mathbf{1}_{\left\{S_{t} \in I\right\}}\left(K-S_{t}\right)^{+}\right] d K d t \\
& +\int_{0}^{T_{P V S}} \int_{\kappa}^{\infty} P_{M}^{\prime \prime}(K) \mathbb{E}\left[\mathbf{1}_{\left\{S_{t} \in I\right\}}\left(S_{t}-K\right)^{+}\right] d K d t
\end{aligned}
$$

for any $\kappa>0$.

Proof: See Appendix D.

\subsection{Hedging Volatility Risk with Polynomial Variance Swap}

In order to improve the problem mentioned in section 2.3, we apply PVS to hedge risk $A$. If a trader can use PVS such that $\mathbf{1}_{\left\{S_{t} \in I\right\}} P_{M}\left(S_{t}\right)=g\left(t, S_{t}\right)$ for all $t \in[0, T]$ and $S_{t}>0$, then risk $A$ is perfectly fixed to $K_{\mathrm{PVS}}$. Moreover, by setting the hedging volatility $\sigma_{\mathrm{H}}=\sigma_{\mathrm{PVS}}$, the initial cost of risk $B$ can be canceled out to the initial payment of PVS $K_{\mathrm{PVS}}$. Similarly to the case of the standard variance swap, there exists a discrepancy between the volatility risk weight and PVS against time $t$ and the underlying asset price $S_{t}$. However, the over/under-hedging problem of PVS is more improvable than that of the standard variance swap. To implement a suitable hedging scheme with PVS, we provide a certain procedure to set up the polynomial as follows.

Fixing a certain time $\tau$, a corridor $I=[a, b]$ and an order $M$ of a polynomial ${ }^{1}$, the coefficients $a_{0}, a_{1}, \cdots, a_{M}$ of the polynomial $P_{M}(x)$ can be determined by solving the least square problem:

$$
\min _{P_{M} \in \mathbf{P}_{M}} \int_{a}^{b}\left\{g(\tau, x)-P_{M}(x)\right\}^{2} d x
$$

\footnotetext{
${ }^{1}$ Although the selection of $\tau, I$, and $M$ appear to be more art than science, it is not so difficult to choose them for effective hedging (see Figure 2).
} 
where $\mathbf{P}_{M}$ is the set of the $M$-th order polynomials. The solution of the problem (3.14) is given by

$$
P_{M}(x)=\sum_{m=0}^{M} b_{m} \phi_{m}(x),
$$

where $\phi_{m}(x)$ is the $m$-th order orthogonal polynomial such that

$$
\int_{a}^{b} \phi_{m}(x) \phi_{n}(x) d x=\left\{\begin{array}{lll}
0 & \text { if } & m \neq k \\
\frac{b-a}{2(m+1)} & \text { if } & m=k
\end{array},\right.
$$

and

$$
b_{m}:=\frac{2(m+1)}{b-a} \int_{a}^{b} \phi_{m}(x) g(\tau, x) d x
$$

Therefore, the coefficients $a_{0}, a_{1}, \cdots, a_{M}$ of $P_{M}(x)$ are determined as real numbers satisfying

$$
\sum_{m=0}^{M} b_{m} \phi_{m}(x)=\sum_{m=0}^{M} a_{m} x^{m} .
$$

For example, Figure 2 plots the volatility risk weights of a call option and a polynomial of PVS. In this example, the 6 -th order PVS with a corridor interval $I=[70,140]$ is applied and the fitting point of the polynomial is set as $\tau=0.5$, while the call option is the same contract as Figure 1. Looking at Figure 2, it can be said that although the mismatching problem cannot be completely overcome $^{2}$, PVS is much better for hedging uncertain volatility risk than the standard variance swap.

When selling a European derivative in Eq.(2.2), the hedging scheme proposed in the above discussion consists of the following positions:

- Hold $\frac{\partial v}{\partial S}\left(t_{n}, S_{t_{n}} ; \sigma_{\mathrm{PVS}}, f^{T}\right)$ units of the underlying asset $S_{t_{n}}$ at each time $t_{n}$ for the Black-Scholes delta hedging.

- Pay the fixed payment $K_{\mathrm{PVS}}$ and receive the realized polynomial variance,

$$
\int_{0}^{T_{\mathrm{PVS}}} \mathbf{1}_{\left\{S_{t} \in I\right\}} P_{M}\left(S_{t}\right) \sigma^{2}(\omega, t) d t,
$$

at maturity of PVS for hedging risk $A$.

- Receive the initial cost of risk $B$,

$$
\sigma_{\mathrm{PVS}}^{2} \int_{0}^{T} v\left(0, S_{0} ; \sigma_{\mathrm{PVS}}, g^{t}\right) d t
$$

and hold

$$
\sigma_{\mathrm{PVS}}^{2} \Delta t \sum_{t_{n}<t_{i}} \frac{\partial v}{\partial S}\left(t_{n}, S_{t_{n}} ; \sigma_{\mathrm{PVS}}, g^{t_{i}}\right),
$$

units of the underlying asset $S_{t_{n}}$ at each time $t_{n}$ for dynamic hedging of risk $B$.

\footnotetext{
${ }^{2}$ As one method to improve the mismatching problem with respect to time $t$, the forward start polynomial variance swaps can be considered as tools of time-piecewise fitting to the volatility risk weight. However, this is not pragmatical because a large number of options are needed for replicating the forward start PVSs.
} 


\section{Simulation Analysis}

This section shows the effectiveness of the hedging scheme proposed in Section 3 under uncertain volatility environments through Monte Carlo simulation tests. To examine the performance of the Black-Scholes delta hedging with PVS, we compare four types of hedging strategies under different three scenarios.

\subsection{Setup of Simulation}

Let us consider the problem faced by the writer of a call option on a certain stock, whose maturity is 3-months and strike is at-the-money. The writer intends to hold this short potion until the maturity, and can hedge his market risk using various hedging schemes. For concreteness, suppose that the initial stock price is $S_{0}=100$, the strike of the target call is $K=100$, and the option maturity is $T=60 / 250$; i.e., we assume that there are 20 business days in a month and 250 business days in a year. Both the interest rate and the dividend yield are set to be zero for simplicity.

The assumed market situation in the simulation tests is as follows: All market options are priced in accordance with the Heston's stochastic volatility model (Heston [1993]) with given parameters, and can be traded without any transaction cost. That is, all traders in the option market believe that the risk-neutral dynamics of the underlying asset price take the following form:

$$
\begin{aligned}
d S_{t} & =\sqrt{V_{t}} S_{t} d W_{t}, \\
d V_{t} & =\xi\left(\eta-V_{t}\right) d t+\theta \sqrt{V_{t}} d Z_{t},
\end{aligned}
$$

where $\xi, \eta$ and $\theta$ are positive constants such that $\xi \eta \geq \theta^{2} / 2$, and $W$ and $Z$ are Brownian motions with correlation $\rho$ under a pricing measure. The writer also knows the fact that the market option prices follow the Heston model through observing the option market. The Heston parameters for the market option prices are listed in Table 1.

Table 1: Heston Parameters for Market Option Prices

\begin{tabular}{ccccc}
\hline$V_{0}$ & $\xi$ & $\eta$ & $\theta$ & $\rho$ \\
\hline $0.20^{2}$ & 1.15 & $0.20^{2}$ & 0.39 & -0.64 \\
\hline
\end{tabular}

On the other hand, the writer does not know the true generating process of the stock price. Thus, we consider the situation that the writer cannot know not only the parameters of the generating model, but also the true model itself.

\subsection{Hedging Strategies}

We employ four hedging strategies in order to compare the performance of our hedging scheme with that of standard hedging schemes. In the simulation tests, the writer carries out one of these strategies systematically to hedge his market risk on the target call until the maturity.

The first strategy is the Black-Scholes delta hedging without PVS (BS DH for short). The writer uses the Black-Scholes model for dynamic hedging of the call option:

$$
d S_{t}=\sigma_{\mathrm{BS}} S_{t} d W_{t}
$$

where $\sigma_{\mathrm{BS}}$ is a constant volatility. At each time $t$, he computes the delta based on the Black-Scholes model and re-balances the dynamic hedging portfolio accordingly. To be comparison with the Black-Scholes delta hedging with PVS, the constant volatility $\sigma_{\mathrm{BS}}$ is set to be the strike volatility of PVS. 
The second strategy is the minimum-variance hedging with the Heston model (HS MVH for short), which is a standard method of dynamic hedging in an incomplete market. In the minimum-variance hedging under the Heston model (4.1), the units of the underlying asset to be held at each time $t$ are computed as follows: ${ }^{3}$

$$
\frac{\partial C_{t}}{\partial S_{t}}+\frac{\rho \theta}{S_{t}} \frac{\partial C_{t}}{\partial V_{t}}
$$

where $C_{t}$ denotes the time- $t$ price of the target call option, and $\rho$ and $\theta$ are parameters in (4.1). Note that volatility risk can be partially hedged through the correlation between the underlying asset price and its instantaneous variance if the model and its parameters are correct. Based on the equation (4.3), the writer re-balances the dynamic hedging portfolio at each time in the simulation tests. For HS MVH, he adopts the same Heston parameters as in Table 1.

The third strategy is the Black-Scholes delta hedging with PVS, in which the PVS maturity is the same as that of the target call; i.e., $T_{\mathrm{PVS} 1}=60 / 250$ (BS DH with PVS1 for short). To hedge uncertain volatility risk on the target call, the writer uses the sixth order PVS with corridor interval $I=[85,120]$. Its polynomial is fitted with the volatility risk weight of the target call at time $\tau_{\mathrm{PVS} 1}=30 / 250$, and the static portfolio replicating the PVS is composed of 4 calls and 4 puts. In order to accurately approximate the static portfolio by the finite number of options, the Gauss-Legendre quadrature rule is applied. More detailed discussion for this approximation scheme can be found in Takahashi and Yamazaki [2009a]. Table 2 reports the static portfolio compositions. At initial time, the writer constitutes the static portfolio for the PVS. Then, he computes the Black-Scholes delta and the amount of the underlying asset for replicating the PVS at each time, and re-balances the dynamic portfolios of both BS DH and the PVS accordingly. Note that, although the writer can roughly offset the volatility risk by using the PVS, there is a discrepancy between the polynomial of the PVS1 and the volatility risk weight of the target call for all time $t \in[0, T]$. In particular, this strategy is considerably over-hedging when time $t$ approaches the target call maturity and the stock price is apart from at-the-money.

Table 2: Static Portfolio for Replicating PVS1

\begin{tabular}{l|rrrr}
\hline & No.1 & No.2 & No.3 & No.4 \\
\hline Call Strike & 101.3886 & 106.6002 & 113.3998 & 118.6114 \\
Call Amount & 0.1906 & 0.2273 & 0.0560 & 0.0089 \\
\hline Put Strike & 86.0415 & 89.9501 & 95.0499 & 98.9585 \\
Put Amount & 0.0180 & 0.1021 & 0.2341 & 0.1488 \\
\hline
\end{tabular}

The fourth strategy is the Black-Scholes delta hedging with PVS, in which the PVS maturity is shorter than that of the target call (BS DH with PVS2 for short). To avoid over-hedging against the volatility risk near the target call maturity, the PVS maturity is set as $T_{\mathrm{PVS} 2}=55 / 250$ in this strategy. Additionally, the fitting point of its polynomial is $\tau_{\mathrm{PVS} 2}=27.5 / 250$. Except for the PVS maturity and the fitting point, the scheme of BS DH with PVS2 is the same as that of BS DH with PVS1. Table 3 shows the static portfolio compositions.

For all strategies, the writer re-balances the dynamic portfolios once a day until the target call maturity in the simulation tests. Then, we monitor the hedging error (profit and loss) of each sample path, which is defined as the

\footnotetext{
${ }^{3}$ For example, see Bakshi et al. [1997] for the detail and for a practical application of the minimum-variance hedging method.
} 
Table 3: Static Portfolio for Replicating PVS2

\begin{tabular}{l|rrrr}
\hline & No.1 & No.2 & No.3 & No.4 \\
\hline Call Strike & 101.3886 & 106.6002 & 113.3998 & 118.6114 \\
Call Amount & 0.1837 & 0.2247 & 0.0622 & 0.0102 \\
\hline Put Strike & 86.0415 & 89.9501 & 95.0499 & 98.9585 \\
Put Amount & 0.0206 & 0.1075 & 0.2292 & 0.1434 \\
\hline
\end{tabular}

difference between the final value of the total hedging portfolio and the payoff of the target call option.

\subsection{Simulation Test}

For the simulation tests, we consider three data generating processes: the Heston model with correct estimated parameters, the Heston model with misspecified parameters, and the CEV process. In each simulation, a time series of a daily underlying asset price is generated according to an Euler-Maruyama approximation of the respective data generating process. Evey simulation result is based upon 10,000 sample paths.

\subsubsection{Simulation under Heston World}

First, we generate underlying asset prices by the Heston model:

$$
\begin{aligned}
d S_{t} & =\mu S_{t} d t+\sqrt{V_{t}} S_{t} d W_{t}^{*} \\
d V_{t} & =\xi\left(\eta^{*}-V_{t}\right) d t+\theta \sqrt{V_{t}} d Z_{t}^{*}
\end{aligned}
$$

where $W^{*}$ and $Z^{*}$ are Brownian motions with correlation $\rho$ under a physical measure. Note that the parameter $\eta^{*}$, which denotes the mean reversion level of the instantaneous variance for the data generating process, is generally smaller than $\eta$ in Eq.(4.1) because of market price of volatility risk. The Heston parameters for generating underlying asset prices are listed in Table 4. This scenario

Table 4: Heston Parameters for Generating Stock Prices

\begin{tabular}{cccccc}
\hline$\mu$ & $V_{0}$ & $\xi$ & $\eta^{*}$ & $\theta$ & $\rho$ \\
\hline 0.06 & $0.20^{2}$ & 1.15 & $0.18^{2}$ & 0.39 & -0.64 \\
\hline
\end{tabular}

indicates that the option market perfectly estimates both the data generating process and its parameters. Such a situation, however, seems to be unrealistic in practice.

Table 5 reports the summary statistics of the Monte Carlo simulation results. Moreover, Figure 3 shows the histograms of hedging errors. In the Heston world with correct estimated parameters, the means of hedging errors are nearly zero for all hedging strategies. Conversely, the standard deviations are very different for each hedging strategy. Since the minimum-variance hedging can partially hedge volatility risk on the target call, the standard deviation of HS MVH is smaller than that of BS DH without PVS. Furthermore, because the PVS can directly hedge the volatility risk, the standard deviations of BS DH with PVS1 and PVS2 are reduced by half from BS DH and HS MVH. In addition, when seeing in detail, the standard deviation of BS DH with PVS2 is smaller than that of BS DH with PVS1. This is the effect of avoiding over-hedging near the target call maturity. From the viewpoint of the standard deviation of the hedging errors, it can be said that BS DH with PVS2 is the best strategy among 
four candidates. However, it is necessary to pay attention to high kurtosis of the hedging errors in BS DH with PVS1 and PVS2. It means that BS DH with PVS has a fat tail distribution of hedging errors. In fact, we can observe fat-tail properties from Figure 3. In particular, the left tail on the histograms is thick; i.e, negative skew. The high kurtosis is caused by the discrepancy between the polynomials of the PVS and the volatility risk weights of the target call with several bad scenarios for BS DH with PVS.

Table 5: Hedge Error under the Heston World

\begin{tabular}{l|rrrr}
\hline Hedging Scheme & BS DH & HS MVH & $\begin{array}{r}\text { BS DH } \\
\text { with PVS1 }\end{array}$ & $\begin{array}{r}\text { BS DH } \\
\text { with PVS2 }\end{array}$ \\
\hline Mean & -0.0045 & -0.0335 & -0.0113 & -0.0137 \\
Std Err & 0.9576 & 0.8685 & 0.4746 & 0.4612 \\
Skewness & -0.7288 & -0.4404 & -1.8565 & -1.7630 \\
Kurtosis & 5.4867 & 3.6180 & 10.8580 & 11.3134 \\
Min & -7.1363 & -5.6175 & -4.3763 & -3.9820 \\
Max & 3.2830 & 2.7374 & 1.5868 & 1.7767 \\
\hline
\end{tabular}

\subsubsection{Simulation under Heston World with Misspecified Parameters}

Second, we generate underlying asset prices by the Heston model (4.4) with misspecified parameters. The misspecified Heston parameters for generating underlying asset prices are listed in Table 6 .

Table 6: Misspecified Heston Parameters for Generating Stock Prices

\begin{tabular}{cccccc}
\hline$\mu$ & $V_{0}$ & $\xi$ & $\eta^{*}$ & $\theta$ & $\rho$ \\
\hline 0.06 & $0.20^{2}$ & 2.00 & $0.25^{2}$ & 0.39 & -0.64 \\
\hline
\end{tabular}

Table 7 reports the basic statistics of the Monte Carlo simulation results, and Figure 4 shows the histograms of hedging errors. Note that BS DH and HS MVH make losses on average while the means of hedging errors of BS DH with PVS1 and PVS2 can be regarded as to be nearly zero. In addition, the standard deviations of BS DH with PVS1 and PVS2 are much smaller than those of BS $\mathrm{DH}$ and HS MVH. That is, by using the PVS the writer can improve the mean of hedging errors as well as the standard deviation. As a result of this simulation test, it can be said that the PVS is an appropriate tool for hedging volatility risk even when the model parameters are mis-estimated, because the PVS does not depend on parameter specification at all. On the other hand, similarly to the previous case, there exists fat-tail risk of the hedging errors in BS DH with PVS.

Table 7: Hedge Error under the Heston World with Misspecified Parameters

\begin{tabular}{l|rrrr}
\hline Hedging Scheme & BS DH & HS MVH & $\begin{array}{r}\text { BS DH } \\
\text { with PVS1 }\end{array}$ & $\begin{array}{r}\text { BS DH } \\
\text { with PVS2 }\end{array}$ \\
\hline Mean & -0.2800 & -0.3131 & -0.0609 & -0.0960 \\
Std Err & 0.9247 & 0.8303 & 0.5302 & 0.4942 \\
Skewness & -1.0346 & -0.5237 & -1.8707 & -2.1345 \\
Kurtosis & 6.0194 & 3.7374 & 9.5258 & 11.1666 \\
Min & -7.1393 & -5.6710 & -4.8121 & -4.3671 \\
Max & 2.6183 & 2.0812 & 1.7729 & 1.4335 \\
\hline
\end{tabular}




\subsubsection{Simulation under CEV World}

Third, we generate underlying asset prices by the CEV model:

$$
d S_{t}=\mu S_{t} d t+\sigma_{\mathrm{CEV}} S_{t}^{\beta} d W_{t}^{*},
$$

where $\beta$ and $\sigma_{\mathrm{CEV}}$ are constant parameters. The CEV parameters for generating underlying asset prices are listed in Table 8. This situation means a model

Table 8: CEV Parameters for Generating Stock Prices

\begin{tabular}{ccc}
\hline$\mu$ & $\beta$ & $\sigma_{\mathrm{CEV}}$ \\
\hline 0.06 & 0.50 & 2.00 \\
\hline
\end{tabular}

misspecification case.

Table 9 reports the basic statistics of the Monte Carlo simulation results, and Figure 5 shows the histograms of hedging errors. Similarly to the misspecified Heston world, although BS DH and HS MVH make losses on average, the means of hedging errors of BS DH with PVS1 and PVS2 are nearly equal to zero. In addition, the standard deviations of BS DH with PVS1 and PVS2 are much smaller than HS MVH. Note that the hedging performance of HS MVH is worse than BS DH without PVS because HS MVH is a fragile hedging scheme for model risk. On the other hand, by adopting BS DH with PVS the writer can improve the mean of hedging errors as well as the standard deviation. As a consequence of this simulation test, it can be also said that BS DH with PVS is a robust hedging scheme for model risk. Of course, fat-tail risk of the hedging errors exists in BS DH with PVS.

Table 9: Hedge Error under the CEV World

\begin{tabular}{l|rrrr}
\hline Hedging Scheme & BS DH & HS MVH & $\begin{array}{r}\text { BS DH } \\
\text { with PVS1 }\end{array}$ & $\begin{array}{r}\text { BS DH } \\
\text { with PVS2 }\end{array}$ \\
\hline Mean & -0.1300 & -0.1702 & -0.0327 & -0.0495 \\
Std Err & 0.4429 & 1.1665 & 0.3866 & 0.3254 \\
Skewness & -0.3633 & -0.0814 & -1.4537 & -1.5130 \\
Kurtosis & 4.7282 & 2.2873 & 6.1156 & 7.4525 \\
Min & -2.5923 & -3.3922 & -2.6899 & -2.7925 \\
Max & 1.8762 & 2.7595 & 0.9525 & 1.1065 \\
\hline
\end{tabular}

\section{Concluding Remarks}

This paper examines how robust the Black-Scholes delta hedging is against uncertain volatility risk when adding a certain variance swap. While the standard variance swap is the most approved contract to purely trade volatility of a underlying asset, it is not absolutely an appropriate tool for hedging uncertain volatility risk on derivative securities. To improve the defect of the standard variance swap, we develop the polynomial variance swap, which is a kind of exotic variance swaps and can be implemented by model-free replication. Then, a new hedging scheme applying PVS is proposed. We test the hedging performance of our scheme through Monte Carlo simulations which generate several different scenarios of the underlying price processes. As a result, it is shown that the hedging scheme proposed in this paper is not perfect, but significantly robust rather than other standard hedging schemes such as the minimum-variance 
hedging. Moreover, it is found that the hedging performance of our scheme is hardly affected by model risk.

Finally, our next research topic will be to consider a robust hedging scheme of exotic derivatives such as barrier options and look-back options against uncertain volatility risk, and design a suitable volatility derivative as a hedging tool for this problem.

\section{A Price and Delta of the Volatility Risk Weight}

We derive the closed-form expressions of $v\left(0, S_{0} ; \sigma_{\mathrm{H}}, g^{t}\right)$ and $\frac{\partial v}{\partial S}\left(0, S_{0} ; \sigma_{\mathrm{H}}, g^{t}\right)$ in Eq.(2.8) when the target derivative is a plain vanilla call. In the cases of other European derivatives such as asset digital and cash digital, the closed-form expressions can be obtained by the same manner as the following discussion.

Consider the payoff function $f^{T}(S)=(S-K)^{+}$with strike $K$. By Eq.(2.5) and the Black-Scholes gamma formula of the call option, the payoff function of the volatility risk weight at maturity $t$ can be written as

$$
g\left(t, S_{t}\right):=g^{t}\left(S_{t}\right)=\frac{1}{2} S_{t} \frac{1}{\sqrt{2 \pi}} \frac{e^{-\frac{1}{2} d_{1}^{2}}}{\sigma_{\mathrm{H}} \sqrt{T-t}},
$$

where

$$
d_{1}=\frac{\ln \frac{S_{t}}{K}+\frac{1}{2} \sigma_{\mathrm{H}}^{2}(T-t)}{\sigma_{\mathrm{H}} \sqrt{T-t}} .
$$

Therefore, the Black-Scholes price, which is the price in the case of $\sigma(\omega, t)=\sigma_{H}$ in Eq.(2.1), of the derivative with payoff $g^{t}$ is given by

$$
\begin{aligned}
v\left(0, S_{0} ; \sigma_{\mathrm{H}}, g^{t}\right) & =\mathbb{E}\left[\frac{1}{2} S_{t} \frac{1}{\sqrt{2 \pi}} \frac{e^{-\frac{1}{2} d_{1}^{2}}}{\sigma_{\mathrm{H}} \sqrt{T-t}}\right] \\
& =\frac{1}{2} S_{0} I\left(S_{0}\right),
\end{aligned}
$$

where

$$
\begin{aligned}
I\left(S_{0}\right) & :=\mathbb{E}\left[\frac{S_{t}}{S_{0}} \frac{1}{\sqrt{2 \pi}} \frac{e^{-\frac{1}{2} d_{1}^{2}}}{\sigma_{\mathrm{H}} \sqrt{T-t}}\right] \\
& =\frac{1}{\sqrt{2 \pi T} \sigma_{\mathrm{H}}} \exp \left\{-\frac{1}{2} \frac{\left(\ln \frac{S_{0}}{K}+\frac{1}{2} \sigma_{\mathrm{H}}^{2} T\right)^{2}}{\sigma_{\mathrm{H}}^{2} T}\right\} .
\end{aligned}
$$

From Eq.(A.3), we have

$$
\begin{aligned}
\frac{\partial v}{\partial S}\left(0, S_{0} ; \sigma_{\mathrm{H}}, g^{t}\right) & =\frac{1}{2}\left[I\left(S_{0}\right)+S_{0} \frac{\partial I}{\partial S}\left(S_{0}\right)\right] \\
& =\left[\frac{1}{4}-\frac{\ln \frac{S_{0}}{K}}{2 \sigma_{\mathrm{H}}^{2} T}\right] I\left(S_{0}\right) .
\end{aligned}
$$

\section{B Proof of Proposition 2}

Let

$$
h(x)=2\left\{x \ln \frac{x^{*}}{\kappa}-x^{*}+\kappa\right\} .
$$


Then

$$
h^{\prime}(x)=2 \ln \frac{x^{*}}{\kappa} \quad \text { and } \quad h^{\prime \prime}(x)=\frac{2}{x} \mathbf{1}_{\{x \in I\}} .
$$

By Itô's formula, we obtain

$$
\frac{1}{2} \int_{0}^{T} h^{\prime \prime}\left(S_{t}\right) S_{t}^{2} \sigma^{2}(\omega, t) d t=h\left(S_{T}\right)-h\left(S_{0}\right)-\int_{0}^{T} h^{\prime}\left(S_{t}\right) d S_{t} .
$$

Since $h(\kappa)=h^{\prime}(\kappa)=0$, we have

$$
\begin{aligned}
h\left(S_{T}\right) & =\int_{0}^{\kappa} h^{\prime \prime}(K)\left(K-S_{T}\right)^{+} d K+\int_{\kappa}^{\infty} h^{\prime \prime}(K)\left(S_{T}-K\right)^{+} d K \\
& =\int_{a}^{\kappa} \frac{2}{K}\left(K-S_{T}\right)^{+} d K+\int_{\kappa}^{b} \frac{2}{K}\left(S_{T}-K\right)^{+} d K .
\end{aligned}
$$

Therefore, by substituting Eq.(B.1), (B.2), and (B.4) into Eq.(B.3), Eq.(3.5) is obtained.

\section{Proof of Proposition 3}

Let

$$
h(x)=\frac{2}{m(m-1)}\left\{(1-m)\left(x^{*}\right)^{m}-m x\left[\kappa^{m-1}-\left(x^{*}\right)^{m-1}\right]-(1-m) \kappa^{m}\right\} .
$$

Then

$$
h^{\prime}(x)=\frac{2}{m-1}\left\{\left(x^{*}\right)^{m-1}-\kappa^{m-1}\right\} \quad \text { and } \quad h^{\prime \prime}(x)=2 x^{m-1} \mathbf{1}_{\{x \in I\}} .
$$

Therefore, by the same discussion as Appendix B, Eq.(3.6) can be obtained.

\section{Proof of Proposition 4}

From the definition of the strike volatility, we have

$$
\sigma_{\mathrm{PVS}}^{2}=\frac{\mathbb{E}\left[\int_{0}^{T_{\mathrm{PVS}}} \mathbf{1}_{\left\{S_{t} \in I\right\}} P_{M}\left(S_{t}\right) \sigma^{2}(\omega, t) d t\right]}{\mathbb{E}\left[\int_{0}^{T_{\mathrm{PVS}}} \mathbf{1}_{\left\{S_{t} \in I\right\}} P_{M}\left(S_{t}\right) d t\right]}=\frac{K_{\mathrm{PVS}}}{L_{\mathrm{PVS}}},
$$

where

$$
L_{\mathrm{PVS}}=\mathbb{E}\left[\int_{0}^{T_{\mathrm{PVS}}} \mathbf{1}_{\left\{S_{t} \in I\right\}} P_{M}\left(S_{t}\right) d t\right] .
$$

Next, for any $\kappa>0$ and all $t \in\left[0, T_{\mathrm{PVS}}\right]$, it satisfies

$$
\begin{aligned}
P_{M}\left(S_{t}\right) & =P_{M}(\kappa)+P_{M}^{\prime}(\kappa)\left(S_{t}-\kappa\right) \\
& +\int_{0}^{\kappa} P_{M}^{\prime \prime}(K)\left(K-S_{t}\right)^{+} d K+\int_{\kappa}^{\infty} P_{M}^{\prime \prime}(K)\left(S_{t}-K\right)^{+} d K .(\mathrm{D} .3)
\end{aligned}
$$

Therefore, by substituting Eq.(D.3) into Eq.(D.2), Eq.(3.13) is obtained. 


\section{Acknowledgment}

The authers are grateful to Mr. Toshifumi Ikemori(Mizuho-DL Financial Technology Co., Ltd.), Mr. Tsuyoshi Hase(Mizuho-DL Financial Technology Co., Ltd.), Mr. Shuji Munekuni(Mizuho-DL Financial Technology Co., Ltd.), Mr. Akira Onuma(Mizuho Corporate Bank, Ltd.), and Mr. Seiichi Tanaka(Mizuho Corporate Bank, Ltd.) for their constant support in implementing this research. The views expressed in the paper are those of the authors and do not necessarily reflect those of Mizuho-DL Financial Technology Co., Ltd. 
Figure 1: Volatility Risk Weight of Call and Variance Swap

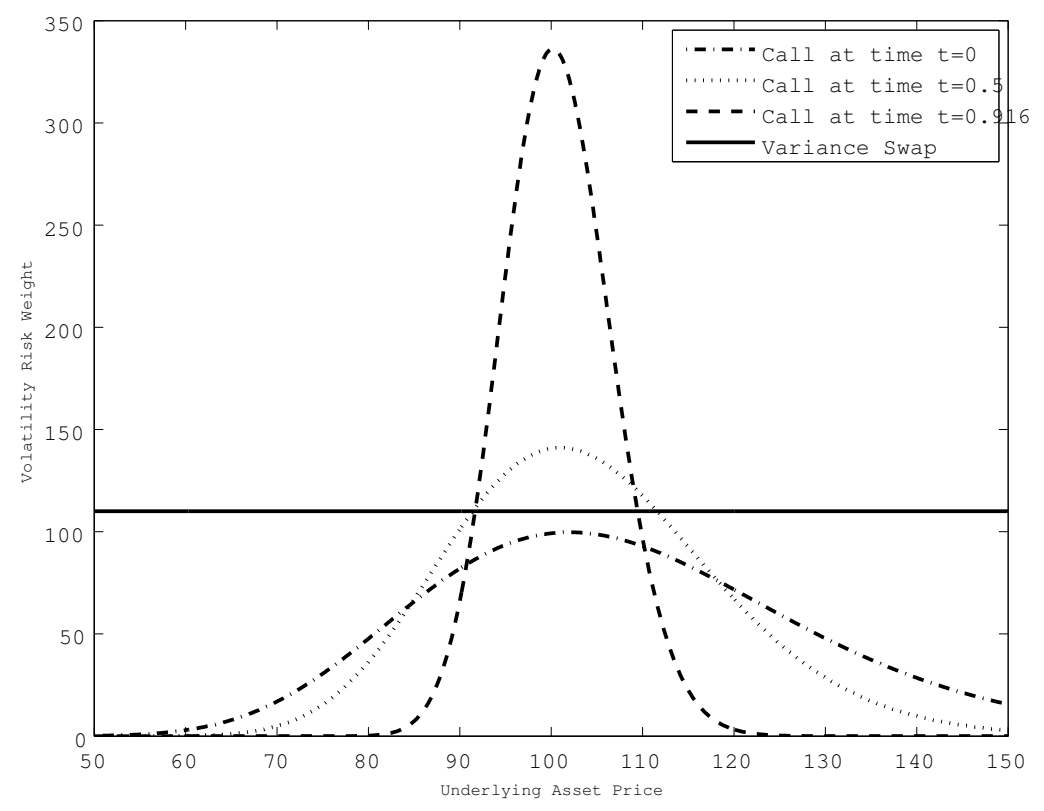

Figure 2: Volatility Risk Weight of Call and Polynomial Variance Swap

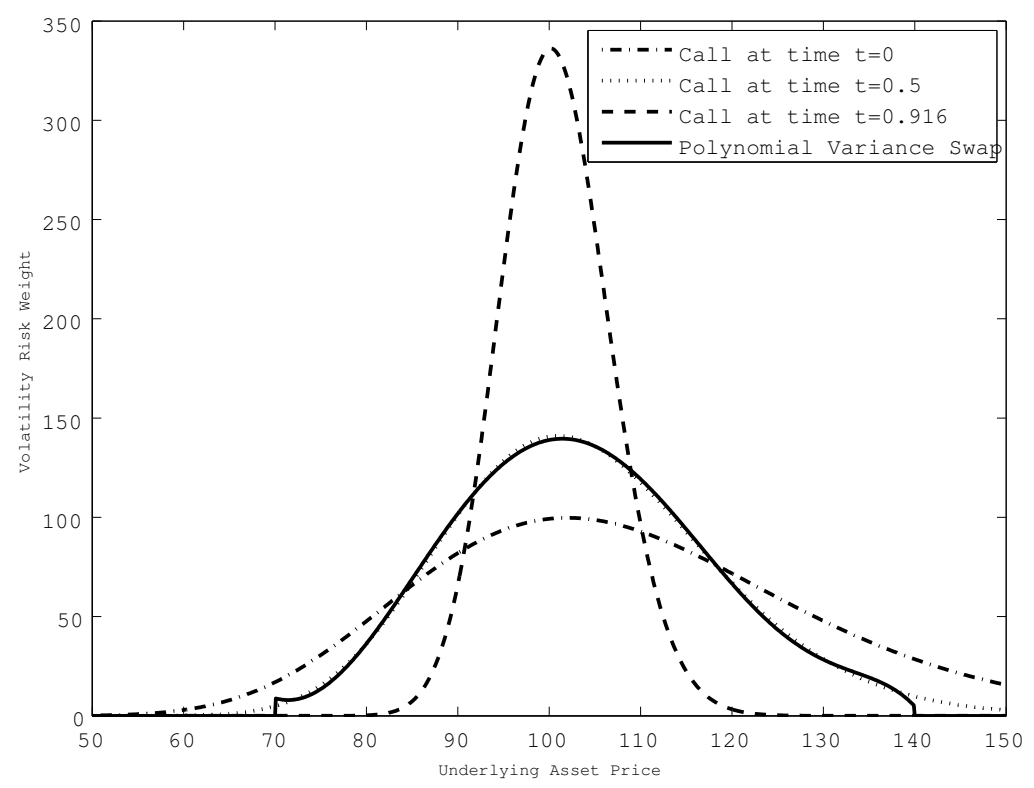




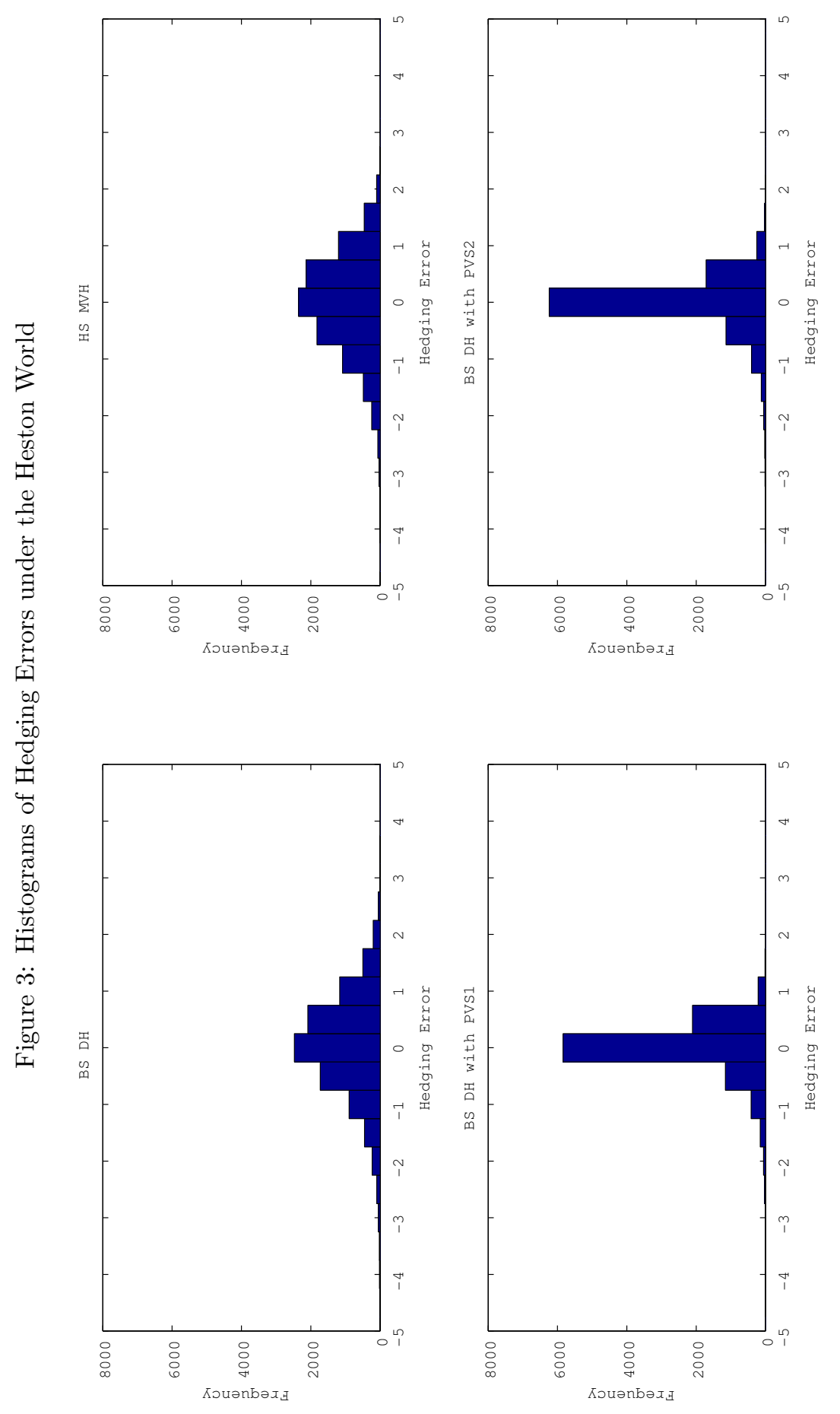




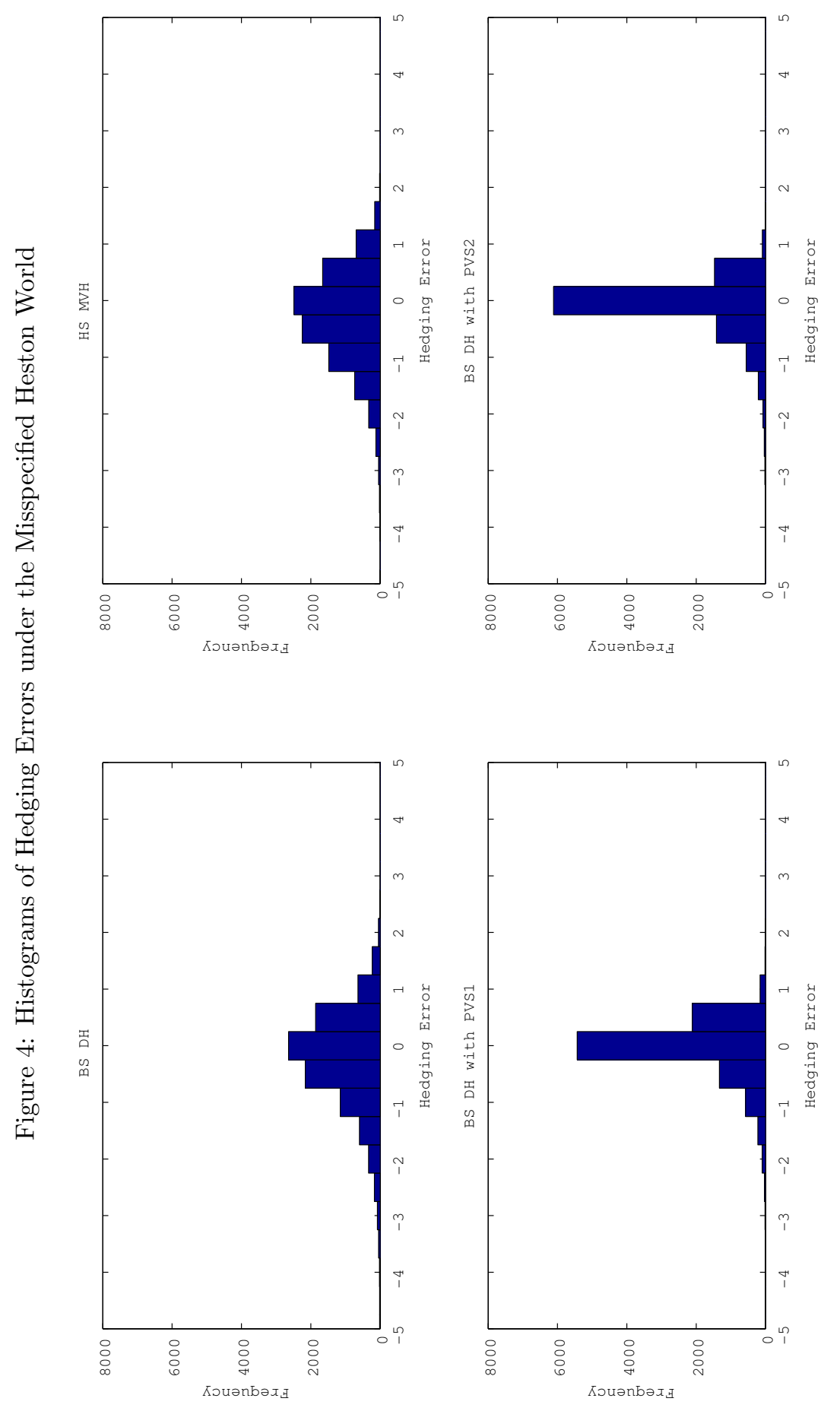




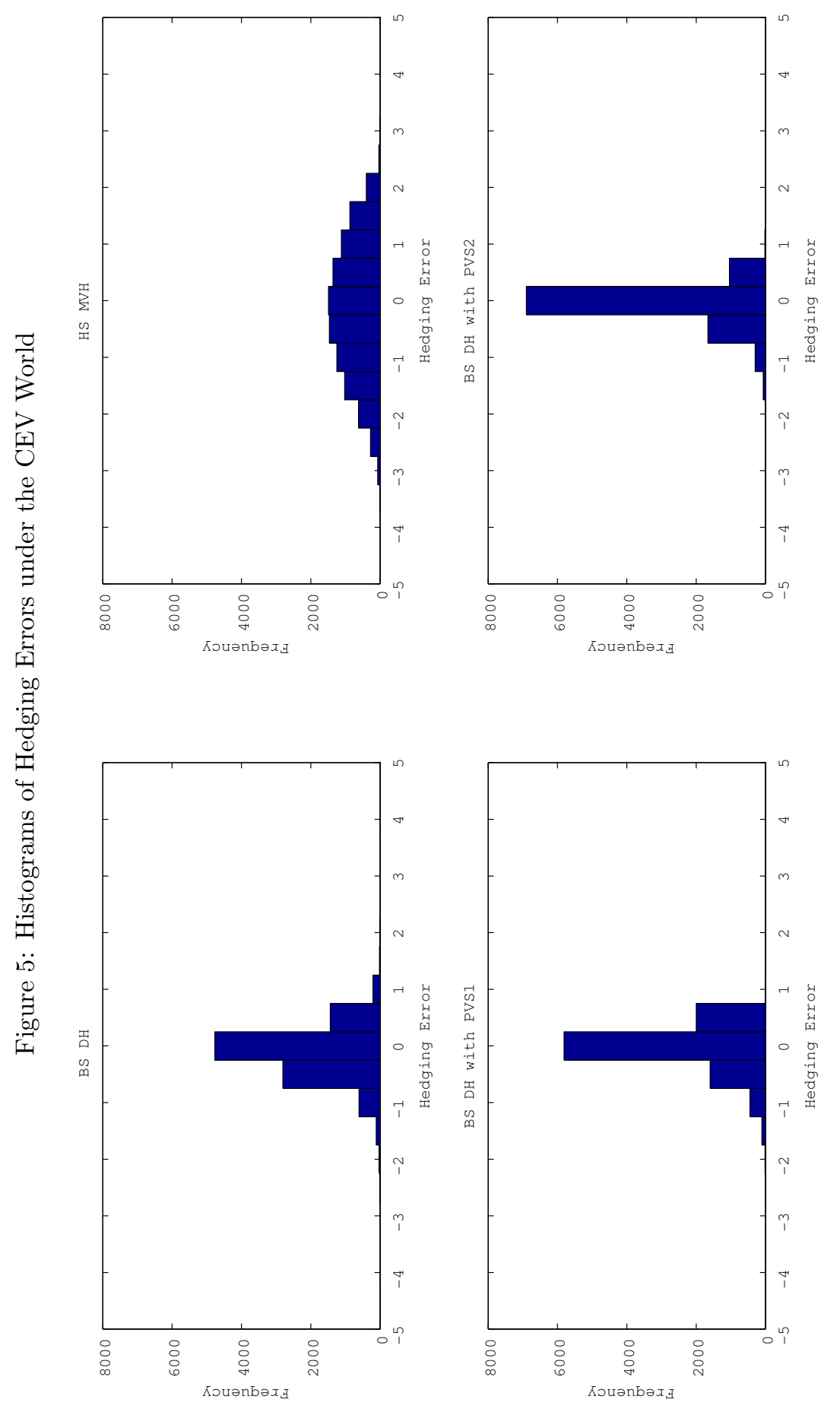

